\title{
Glycodelin As A Biomarker Of Advanced Lung Adenocarcinoma Brain Metastases In Patients Treated With EGFR Tyrosine Kinase Inhibitors
}

This article was published in the following Dove Press journal:

Cancer Management and Research

\author{
Zexu $\mathrm{Ni}^{1}{ }^{1} *$ \\ Lingling Zhang ${ }^{2,3, *}$ \\ Jinhua Zheng' \\ Xiaojie Su' \\ Shucai Zhang ${ }^{2}$ \\ 'Department of Pathology, Dongguan \\ Tungwah Hospital, Dongguan 523II0, \\ People's Republic of China; ${ }^{2}$ Department \\ of Oncology, Beijing Chest Hospital, \\ Capital Medical University; Beijing \\ Tuberculosis and Thoracic Tumor \\ Research Institute, Beijing 10II49, \\ People's Republic of China; ${ }^{3}$ Department \\ of Oncology, Peking University \\ International Hospital, Beijing 102206, \\ People's Republic of China \\ *These authors contributed equally to \\ this work
}

Correspondence: Shucai Zhang Department of Oncology, Beijing Chest Hospital, Capital Medical University; Beijing Tuberculosis and Thoracic Tumor Research Institute, Beijing I0II49, People's Republic of China Email sczhang6304@163.com
Objective: Brain metastasis (BM) is a serious complication of advanced lung adenocarcinoma and is a prominent factor leading to lung cancer mortality. In this study, the expression of the glycodelin protein was analyzed in EGFR-mutant tyrosine kinase inhibitor-sensitive advanced lung adenocarcinoma.

Methods: This study features a retrospective analysis of 74 advanced lung adenocarcinoma patients treated at our hospital from January 2010 to December 2017. The expressions of glycodelin were assessed by standard immunohistochemistry and correlated with clinicopathological factors and overall survival (OS) outcomes.

Results: Patients with advanced lung adenocarcinoma with glycodelin overexpression were prone to BM $(\mathrm{P}<0.05)$, and exhibited significantly shortened OS (11.8 months vs 20.4 months, $\mathrm{P}<0.05)$. Multivariate regression analysis showed that overexpression of glycodelin and brain metastases were independent factors affecting the prognosis of advanced lung adenocarcinoma $(\mathrm{P}<0.05)$.

Conclusion: The overexpression of glycodelin is closely related to the presence of brain metastasis in lung adenocarcinoma, and can be used as an auxiliary diagnostic index for prognosis of advanced lung adenocarcinoma.

Keywords: glycodelin, advanced lung adenocarcinoma, brain metastasis, prognosis

\section{Introduction}

Lung cancer is the leading cause of cancer death worldwide, and its morbidity and mortality are rapidly increasing. ${ }^{1}$ Non-small-cell lung cancer (NSCLC) accounts for approximately $85 \%$ of lung cancer cases. ${ }^{2}$ Lung adenocarcinoma with epidermal growth factor (EGFR) gene mutations can benefit significantly from EGFR tyrosine kinase inhibitors (TKI), which have become the first-line therapy for patients harboring such cancers. However, prognosis for patients with advanced lung adenocarcinoma is still poor, especially for patients with brain metastases (BM). The median overall survival of lung adenocarcinoma patients with BM is approximately 9.3-19.1 months. $^{3,4}$ The development of clinically useful prognostic molecular markers is crucial to identify subsets of advanced lung adenocarcinoma patients with poor survival outcomes.

Glycodelin is a protein that has been well described during the menstrual cycle and pregnancy. ${ }^{5,6}$ Besides its function during pregnancy and implantation, glycodelin is overexpressed in hormone-related cancers, such as ovarian cancer and breast cancer. ${ }^{7,8}$ Additionally, glycodelin is also expressed in melanoma, malignant 
pleural mesothelioma, and NSCLC. ${ }^{9,10}$ The prognostic value of glycodelin expression in NSCLC has been previously described, ${ }^{11}$ but whether its expression is associated with clinicopathologic features like BM and EGFR mutation has not been reported.

The aim of this study was to evaluate glycodelin expression levels in advanced lung adenocarcinoma by standard immunohistochemistry (IHC) in order to interrogate potential associations between glycodelin expression and brain metastasis and poor survival outcome.

\section{Methods}

\section{Patients}

The clinical study was reviewed and approved by Medical Ethics Committee of Donghua Hospital of Dongguan (Guangdong, China) and informed consent forms were signed by all patients or guardians. We retrospectively collected clinical information from 74 patients with histologically confirmed metastatic lung adenocarcinoma, treated at the Donghua Hospital of Dongguan from January 2010 to December 2017. We examined patients whose cancers harbored activating EGFR mutation (exon 19 deletion or exon 21 L858R mutation) who were treated with EGFR-TKI in the first or second-line setting. The inclusion criteria were as follows: patients diagnosed as stage IV pulmonary adenocarcinoma by bronchus or CT-guided biopsy; EGFR exon 19 or 21 activating mutation present, and patient received EGFR-TKIs during the treatment; Eastern Cooperative Oncology Group performance status (ECOG) was 0-2; function of the bone marrow, heart, liver, kidney and other organs was not abnormal; lesions measured by imaging; survival period longer than 3 months; and a brain enhancement MRI was performed before treatment. Patients with arteriovenous thrombosis, active infection, cardiovascular infarction, stroke, and severe trauma were excluded.

\section{Immunohistochemistry (IHC)}

Before the first-line therapy, tumor tissue samples were obtained for immunohistochemical (IHC) evaluation of glycodelin overexpression, as previously described. ${ }^{12}$ After incubation in methanol/ $\mathrm{H}_{2} \mathrm{O}_{2}$ for $30 \mathrm{~min}$, sections were washed in phosphate buffered saline (PBS), then treated with goat serum (Vectastain ${ }^{\circledR}$ ABC kit, Vector Laboratories, Burlingame, CA, USA). Incubation with polyclonal anti-glycodelin antibody (sc-12289, Santa Cruz Biotechnology, Heidelberg, Germany) was performed overnight at $4^{\circ} \mathrm{C}$. Sections were then incubated with biotinylated secondary goat-anti-rabbit antibody (Vectastain ${ }^{\circledR} \mathrm{ABC}$ kit) and avidin-biotinylated peroxidase (Vectastain ${ }^{\circledR} \mathrm{ABC}$ kit). Peroxidase staining reaction was performed with $1 \mathrm{mg} / \mathrm{mL}$ diaminobenzidine $/ \mathrm{H}_{2} \mathrm{O}_{2}$ for $5 \mathrm{~min}$, and the reaction was stopped with tap water. Sections were counterstained with hematoxylin and then cover-slipped. For controls, the primary antibody was replaced with pre-immune rabbit serum. Staining was observed with an Olympus BX51 microscope (Olympus Corporation, Tokyo, Japan).

\section{IHC Evaluation}

Glycodelin staining was mainly observed in the cytoplasm, and positively stained cells were brown or yellow. The immunostaining was evaluated with a semi-quantitative scoring system based on a combination of staining intensity and the proportion of the stained tumor cells. ${ }^{13}$ The immunoreactive score (values $0-9$ ) was calculated by multiplying the signal intensity (negative $=0$; weakly positive $=1$; moderate positive $=2$; strong positive $=3$ ) and the percentage of positive tumor cells $(<10 \%=1 ; 10-50 \%=2 ;>50 \%=3)$. An immunoreactive score of 4 was determined to be the cutoff point to consider glycodelin overexpression. Stained sections were evaluated independently by two pathologists who were blind to patient information. If the results were different, a third pathologist would evaluate for confirmation.

\section{Follow Up}

All patients were followed up from the initial treatment though December 2018, using outpatient review or telephone follow-up. The routine items informed by outpatient reexamination include craniocerebral MRI, chest CT, upper abdominal CT, radionuclide bone imaging, and superficial lymph node ultrasonography. Overall survival (OS) was the time from initial treatment to death or last follow-up.

\section{Statistical Analysis}

Statistical analysis was performed using SPSS 19.0 statistical software (SPSS Inc., Chicago, IL, USA). Correlation between overexpression and clinicopathological characteristics was evaluated by the Pearson Chi-square test. Survival curves were plotted using the Kaplan-Meier method and differences in survival rate were assessed using the log rank test. Univariate and multivariate analysis of prognostic factors was performed using Cox proportional hazards modeling. A two-sided P-value $<0.05$ was considered statistically significant. 


\section{Results}

\section{Glycodelin Expression And} Clinicopathological Features

Overall, 40 patients were female, and the mean patient age was 55.1 years $( \pm 10.2)$. Fifteen patients developed brain metastasis. We found that $40.5 \%(30 / 74)$ of the advanced lung adenocarcinoma patients exhibited strong glycodelin expression by immunohistochemical staining (Figure 1). Further histopathological analysis (Table 1) showed that glycodelin overexpression was associated with age, and the risk of brain metastasis in patients with overexpression of glycodelin was significantly increased $(\mathrm{P}<0.05)$. There were no significant differences in glycodelin expression associated with smoking status or gender $(\mathrm{P}>0.05)$.

\section{Glycodelin Overexpression And Prognosis}

OS in the glycodelin overexpression group was 11.8 months (95\% CI 9.8-13.8 months), and was significantly shorter compared to 20.5 months in the glycodelin negative group (95\% CI 15.8-24.1 months; $\mathrm{P}<0.001$; Figure 2). We performed multivariate Cox model analyses to determine whether glycodelin and clinicopathological factors are independent predictors of OS outcome in patients with advanced lung adenocarcinoma. We found that over expression of glycodelin (HR 3.02, 95\% CI 1.68-4.80, P < 0.001) and brain metastasis (HR $0.32,95 \%$ CI $0.11-3.2, \mathrm{P}=0.04$ ) were both independent factors significantly correlated with poor OS for patients with advanced lung adenocarcinoma (Table 2).

\section{Discussion}

Glycodelin is overexpressed in several kinds of cancer, including NSCLC. According to previous reports, glycodelin overexpressed in more than $75 \%$ of lung adenocarcinoma. ${ }^{10,11}$ In this study, the expression levels of glycodelin were significantly overexpressed in $40.5 \%$ of lung adenocarcinomas, which is lower than previously reported. This may be due to the high cutoff value chosen to indicate overexpression, and the patient cohort in our study is larger than in previous reports. Additionally, ethnic differences may also affect the expression of glycodelin. We found that overexpression of glycodelin is associated with age and brain metastasis, and was not significantly associated with smoking status, gender, or EGFR mutation subtype; these results need to be further confirmed in a larger study population.

These data demonstrate that glycodelin may be a valuable biomarker for NSCLC diagnosis, monitoring, and prognosis. It has been reported that glycodelin expression is a poor prognostic factor for NSCLC patients, especially for female patients, ${ }^{11,12}$ our study yielded a similar result, as the OS for adenocarcinoma patients treated with EGFRTKIs was 11.8 months in the glycodelin overexpression group and 20.5 months in the glycodelin negative group. This difference in survival may be due to the immunomodulatory functions of glycodelin. Previous reports indicated that higher glycodelin expression may increase immune system suppression and promote the metastatic spread of tumor cells. ${ }^{11}$ Glycodelin overexpression has been associated with the activation of a number of signaling pathways, and glycodelin expression in lung cancer cell lines was associated with the transforming growth factor (TGF)- $\beta$ pathway, the phosphoinositide 3-kinase $(\mathrm{PI} \mathrm{K}) /$ protein kinase B (AKT) pathway, and the mitogen-activated protein kinase kinase (MEK)/extracellularsignal regulated kinases (ERK) pathway, ${ }^{14}$ these data need to be further confirmed in vivo.

In this study, we discovered that the risk of brain metastasis was increased in patients with advanced lung
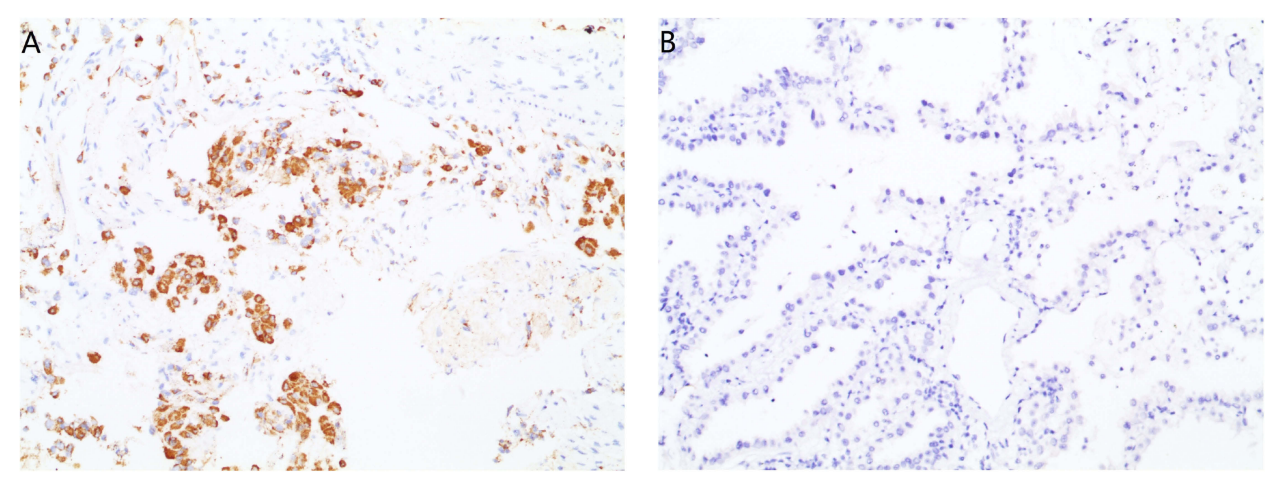

Figure I Positive (A) and negative (B) Glycodelin protein expression by immunohistochemistry in advanced lung adenocarcinoma (original magnification 200x). 
Table I Relationship Between Glycodelin Protein Expression And Clinicopathological Characteristics In Advanced Adenocarcinoma Patients $(n=74)$

\begin{tabular}{|c|l|l|l|}
\hline \multirow{2}{*}{ Factors } & \multicolumn{2}{|l|}{$\begin{array}{l}\text { Glycodelin Protein } \\
\text { Expression }\end{array}$} & \\
\cline { 2 - 3 } & Negative & Positive & \\
\hline $\begin{array}{l}\text { Age(years) } \\
<60 \\
\geq 60\end{array}$ & 33 & 12 & \\
\hline $\begin{array}{l}\text { Gender } \\
\text { Male }\end{array}$ & 11 & 18 & $<0.05^{*}$ \\
Female & 19 & 15 & \\
\hline $\begin{array}{l}\text { History of smoking } \\
\text { Never } \\
\text { Smoker }\end{array}$ & 25 & 15 & 0.56 \\
\hline $\begin{array}{c}\text { ECOG PS } \\
\text { 0-I } \\
2\end{array}$ & 28 & 21 & \\
\hline $\begin{array}{c}\text { Brain metastasis } \\
\text { Absent } \\
\text { Present }\end{array}$ & 16 & 9 & 0.57 \\
\hline $\begin{array}{l}\text { EGFR mutation subtype } \\
\text { Exon I9 del } \\
\text { L858R mutation }\end{array}$ & 20 & 19 & 0.13 \\
\hline
\end{tabular}

Note: $* P<0.05$ was considered statistically significant.

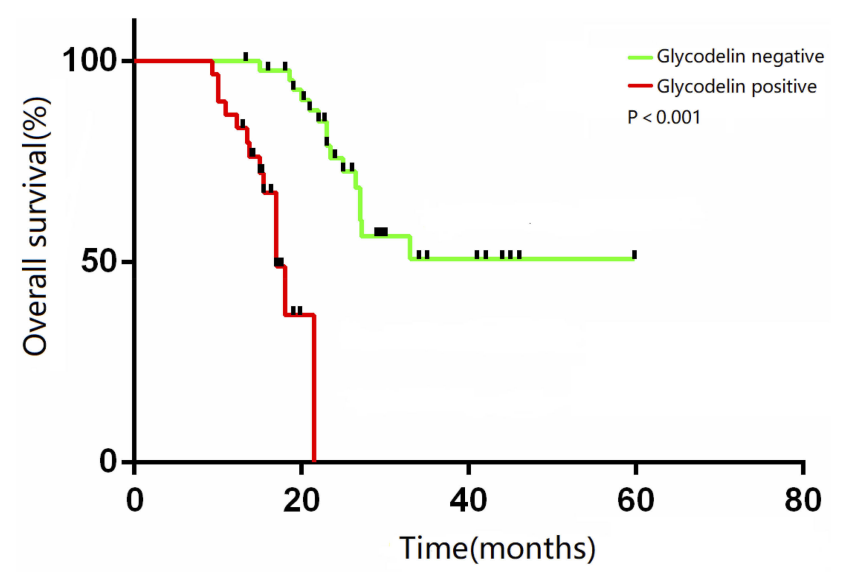

Figure 2 Kaplan-Meier curves of overall survival in advanced lung adenocarcinoma patients.

adenocarcinoma harboring glycodelin overexpression. NSCLC BM patients often have poor prognosis, with a median overall survival time of only 7 months. ${ }^{15}$ The median survival time of BM patients with tumoral EGFR mutations is more favorable, and ranges from 19 to 26 months, ${ }^{4,16}$ though slightly improved, this poor survival
Table 2 Multivariate Cox Regression Analysis Of Factors Possibly In Influencing Overall Survival In Patients With Advanced NSCLC $(n=74)$

\begin{tabular}{|l|l|l|}
\hline Variable & HR $(95 \%$ CI $)$ & P-value \\
\hline $\begin{array}{l}\text { Gender } \\
\text { Male } \\
\text { Female }\end{array}$ & $\mathrm{I}$ & \\
\hline $\begin{array}{l}\text { Age(years) } \\
<60 \\
\geq 60\end{array}$ & $0.83(0.22-2.15)$ & 0.78 \\
\hline $\begin{array}{l}\text { ECOG PS } \\
\text { I } \\
\geq 2\end{array}$ & $\mathrm{I}$ & \\
\hline $\begin{array}{l}\text { History of moking } \\
\text { Never } \\
\text { Smoker }\end{array}$ & $\mathrm{I} .16(0.47-2.88)$ & 0.59 \\
\hline $\begin{array}{l}\text { Brain metastasis } \\
\text { Presen } \\
\text { Absent }\end{array}$ & $1.37(0.55-2.82)$ & 0.48 \\
\hline $\begin{array}{l}\text { Glycodelin protein expression } \\
\text { Negative } \\
\text { Positive }\end{array}$ & $\mathrm{I}$ & \\
\hline
\end{tabular}

Notes: $\mathrm{P}<0.05$ marked with*; $\mathrm{P}<0.001$ marked with**.

time remains unsatisfactory. All of the patients in our study were treated with first generation EGFR-TKI drugs; these first-generation drugs are inefficient at penetrating the blood-brain barrier, and cannot control brain metastatic disease, which ultimately leads to patient mortality. Targeting glycodelin might be a new therapeutic strategy for the treatment of NSCLC BM patients. Whether glycodelin overexpression directly affects the sensitivity to first generation TKI drugs remains to be confirmed. In addition, it remains to be determined whether glycodelin expression is important in the prognosis of patients treated with oxetinib, a third-generation TKI drug that can efficiently penetrate the blood-brain barrier.

Our study had several limitations. First, this was a retrospective study, not a prospective study. Second, there are inherent methodological limitations and variations in the performance and scoring of standard IHC analysis and results, such as were used in this study. Furthermore, additional studies with a larger clinical sample cohort from different centers would be of value to further validate our results. Our results require further confirmation in a larger number of advance lung adenocarcinoma cases. 
In summary, this study demonstrated that glycodelin overexpression is an independent prognostic factor for poor overall survival in advanced lung adenocarcinoma patients with EGFR-TKI sensitive mutations. Moreover, the risk of brain metastasis is increased in patients with glycodelin overexpression. Further study is warranted to determine if interfering with the protein functions of glycodelin improves patient survival.

\section{Acknowledgments}

Lingling Zhang is currently working in the Department of Oncology, Peking University International Hospital, Beijing 102206, People's Republic of China Zexu Ni and Lingling Zhang are co-first authors for this study.

\section{Disclosure}

The authors report no conflicts of interest in this work.

\section{References}

1. Siegel RL, Miller KD, Jemal A. Cancer statistics, 2018. CA Cancer J Clin. 2018;68(1):7-30. doi:10.3322/caac.21442

2. Ettinger DS, Akerley W, Borghaei H, et al. Non-small cell lung cancer. JNCCN. 2012;10(10):1236-1271.

3. Baek MY, Ahn HK, Park KR, et al. Epidermal growth factor receptor mutation and pattern of brain metastasis in patients with non-small cell lung cancer. Korean J Intern Med. 2018;33(1):168-175. doi:10.3904/ kjim. 2015.158

4. Welsh JW, Komaki R, Amini A, et al. Phase II trial of erlotinib plus concurrent whole-brain radiation therapy for patients with brain metastases from non-small-cell lung cancer. J Clin Oncol. 2013;31(7):895902. doi:10.1200/JCO.2011.40.1174

5. Bersinger NA, Birkhauser MH, Yared M, Wunder DM. Serum glycodelin pattern during the menstrual cycle in healthy young women. Acta Obstet Gynecol Scand. 2009;88(11):1215-1221. doi:10.3109/ 00016340903294264
6. Alok A, Karande AA. The role of glycodelin as an immune-modulating agent at the feto-maternal interface. J Reprod Immunol. 2009;83 (1-2):124-127. doi:10.1016/j.jri.2009.06.261

7. Mandelin E, Lassus H, Seppala M, et al. Glycodelin in ovarian serous carcinoma: association with differentiation and survival. Cancer Res. 2003;63(19):6258-6264.

8. Hautala LC, Greco D, Koistinen R, et al. Glycodelin expression associates with differential tumour phenotype and outcome in sporadic and familial non-BRCA1/2 breast cancer patients. Breast Cancer Res Treat. 2011;128(1):85-95. doi:10.1007/s10549-010-1065-y

9. Schneider MA, Muley T, Kahn NC, et al. Glycodelin is a potential novel follow-up biomarker for malignant pleural mesothelioma. Oncotarget. 2016;7(44):71285-71297. doi:10.18632/oncotarget. 12474

10. Schneider MA, Granzow M, Warth A, et al. Glycodelin: a new biomarker with immunomodulatory functions in non-small cell lung cancer. Clin Cancer Res. 2015;21(15):3529-3540. doi:10.1158/10780432.CCR-14-2464

11. Schneider MA, Muley T, Weber R, et al. Glycodelin as a serum and tissue biomarker for metastatic and advanced NSCLC. Cancers 2018;10(12). doi:10.3390/cancers 10110400 .

12. Kunert-Keil C, Steinmuller F, Jeschke U, Gredes T, Gedrange T. Immunolocalization of glycodelin in human adenocarcinoma of the lung, squamous cell carcinoma of the lung and lung metastases of colonic adenocarcinoma. Acta Histochem. 2011;113(8):798-802. doi:10.1016/j.acthis.2010.11.009

13. Spano JP, Andre F, Morat L, et al. Chemokine receptor CXCR4 and early-stage non-small cell lung cancer: pattern of expression and correlation with outcome. Ann Oncol. 2004;15(4):613-617. doi:10.1093/ annonc/mdh 136

14. Weber R, Meister M, Muley T, et al. Pathways regulating the expression of the immunomodulatory protein glycodelin in nonsmall cell lung cancer. Int $J$ Oncol. 2019;54(2):515-526. doi:10.3892/ijo.2018.4654

15. Zimmermann S, Dziadziuszko R, Peters S. Indications and limitations of chemotherapy and targeted agents in non-small cell lung cancer brain metastases. Cancer Treat Rev. 2014;40(6):716-722. doi:10.1016/j.ctrv.2014.03.005

16. Jiang T, Su C, Li X, et al. EGFR TKIs plus WBRT demonstrated no survival benefit other than that of TKIs alone in patients with NSCLC and EGFR mutation and brain metastases. J Thorac Oncol. 2016;11 (10):1718-1728. doi:10.1016/j.jtho.2016.05.013
Cancer Management and Research

\section{Publish your work in this journal}

Cancer Management and Research is an international, peer-reviewed open access journal focusing on cancer research and the optimal use of preventative and integrated treatment interventions to achieve improved outcomes, enhanced survival and quality of life for the cancer patient.
The manuscript management system is completely online and includes a very quick and fair peer-review system, which is all easy to use. Visit http://www.dovepress.com/testimonials.php to read real quotes from published authors. 